\title{
Do we need to adopt antifungal stewardship programmes?
}

\author{
Konstantinos Ioannidis, ${ }^{1}$ Apostolos Papachristos, ${ }^{1}$ Ioannis Skarlatinis, ${ }^{1}$ \\ Fevronia Kiospe, ${ }^{2}$ Sotiria Sotiriou, ${ }^{2}$ Eleni Papadogeorgaki, ${ }_{1}^{3}$ George Plakias, ${ }^{3}$ \\ Vangelis D Karalis, ${ }^{4}$ Sophia L Markantonis ${ }^{2}$
}

\begin{abstract}
${ }^{1}$ Department of Clinical Pharmacy, Diagnostiko kai Therapeftiko Kedro Athinas Hygeia, Athens, Greece ${ }^{2}$ National and Kapodistrian University of Athens Faculty of Pharmacy, Athens, Greece ${ }^{3}$ Department of Microbiology, Diagnostiko kai Therapeftiko Kedro Athinas - Hygeia, Athens, Greece

${ }^{4}$ Faculty of Pharmacy, Laboratory of Biopharmaceutics - Pharmacokinetics, National and Kapodistrian University of Athens, Athens, Greece
\end{abstract}

Correspondence to Dr Konstantinos loannidis, Department of Clinical Pharmacy, Hygeia Hospital Athens, Marousi 151 23, Greece; kioannidis@hygeia.gr

Received 15 December 2017 Revised 22 March 2018 Accepted 22 May 2018 Published Online First 28 June 2018

EAHP Statement 4: Clinical Pharmacy Services.

\begin{abstract}
Background Although antimicrobial stewardship programmes are one of the highest priorities in healthcare systems and many articles have been published, few refer to the implementation of antifungal stewardship and highlight specific points on which efforts should be focused.

Objective To assess the percentage of patients with confirmed candidaemia in whom de-escalation was conducted, and the economic impact of step-down or step-up antifungal therapy. Additionally, we attempted to estimate the potential increase in drug minimum inhibitory concentrations or to detect resistant strains of Candida species.
\end{abstract}

Methods We selected, retrospectively, patients who had received systemic antifungal therapy between 2011 and 2016 for documented candidaemia. Statistical analysis and diagrams were used to assess the results.

Results Of 157 patients with confirmed candidaemia, 58 received azoles, 74 echinocandinsand 18 liposomal amphotericin B for empirical therapy. 51 patients were eligible to step-down to fluconazole but only 23 patients did so. Furthermore, in nine patients unjustified step-up from fluconazole to echinocandins or liposomal amphotericin B was carried out. The additional cost incurred bythe healthcare system due to high prices of echinocandins and liposomal amphotericin B in comparison with fluconazole was $€ 211$ 837. Interestingly, it was found that one strain of $C$. albicans and two strains of $C$. glabrata were resistant to echinocandins.

Conclusion The presence of a multidisciplinary team, including an infection control specialist and a clinical pharmacist, would limit the prescription of advanced antifungal agents as empirical therapy. Moreover, this team would control the de-escalation process - where applicable-leading to a reduction in costs and, probably, a decrease in the emergence of resistant Candida species. These facts contribute to the broader discussion on the adoption of antifungal stewardship programmes.

\section{INTRODUCTION}

The term candidaemia is used to describe the presence of Candida species in the blood. The presence of Candida in a blood culture should always prompt a search for the source of the infection. ${ }^{1}$ Clinical manifestations of infection with Candida species range from local mucous membrane infections to widespread dissemination with multisystem organ failure. ${ }^{2}$ It has often been found in immunocompromised patients and in critically ill patients in intensive care units (ICUs). Candida albicans is the most common cause of candidaemia, but increasingly other Candida species, such as C. parapsilosis, C. glabrata, C. tropicalis and C. krusei, have been isolated. $^{3}$

Treatments for candidaemia include azoles (fluconazole, voriconazole), polyenes (liposomal amphotericin B) and echinocandins (anidulafungin, micafungin, caspofungin). The latest guidelines suggest that the initial antifungal agent should be an echinocandin. ${ }^{2}$ However, breakthrough infections from resistant Candida species have led to speculation about their unnecessary use, their duration of use and their use as prophylactic agents. ${ }^{4}$ Moreover, both echinocandins and liposomal amphotericin B are expensive. ${ }^{5}$ Thus, treatment should be de-escalated to fluconazole both in neutropenic and non-neutropenic patients, when possible. ${ }^{6}$ This is known as stepdown therapy and can be used to reduce the cost of treatment as fluconazole is inexpensive. De-escalation is not recommended for haemodynamically unstable patients, for patients suspected to have endocarditis and for those with species resistant to fluconazole. ${ }^{7}$ Deviation from the guidelines occurs frequently, ${ }^{8}$ because physicians do not believe that de-escalation will work. ${ }^{9}$

The aim of is study is:

- To examine the distribution of Candida species.

- To observe a potential increase in the minimum inhibitory concentration (MICs) of echinocandins and liposomal amphotericin B.

- To assess the percentage of patients for whom antifungal treatment was de-escalated after identification of the susceptibility of the strain according to the guidelines of antifungal therapy.

- To calculate the financial cost, in those cases where the patient met the criteria for de-escalation from echinocandins to fluconazole or for continuing treatment with fluconazole but this was not carried out.

\section{METHODS}

A retrospective analysis (2011-2016) of the clinical data of patients with confirmed candidaemia was performed. Data were obtained from patients records, the microbiology laboratory and the pharmacy department. Patients were screened according to the following criteria:
Papachristos A, Skarlatinis I, et al. Eur J Hosp Pharm 2020;27:14-18. 


\section{Inclusion criteria}

- Patients aged $>18$ years.

- Candidaemia confirmed with blood cultures positive for Candida species.

- Empirical treatment with echinocandin or liposomal amphotericin B until culture results were obtained.

- Strain of Candida spp susceptible to fluconazole.

- Repeated blood cultures not positive for Candida spp

- No administration of vasopressors (epinephrine, norepinephrine, phenylephrine).

\section{Exclusion criteria}

- Age $\leq 18$ years old.

- Diagnosis of endocarditis.

- Strain resistant to fluconazole.

- Contraindications for fluconazole administration.

- Repeated blood cultures positive for Candida spp.

- Administration of vasopressors (epinephrine, norepinephrine, phenylephrine).

The study was conducted at Hygeia Hospital in Athens, Greece, which is a private 280-bed general hospital, where an antimicrobial stewardship programme is in place, but antifungal agents are not used. The study was approved by the scientific and ethical committees of the hospital. Demographic characteristics, such as age, gender, comorbidities and duration of therapy, were recorded. The original cause of hospitalisation was documented and a search made for endocarditis and neutropenia; use of chemotherapy or radiotherapy; and administration of vasopressors. Twenty-eight day mortality was recorded. The defined daily dose (DDD) per 100 patient-days of echinocandins and liposomal amphotericin B was also documented. The study was based on blood cultures positive for Candida spp and MICs for each case. The MICs of each drug were defined by the revised Clinical and Laboratory Standards Institute (CLSI) antifungal breakpoints for resistance. ${ }^{10}$

\section{Statistical analysis}

Results were presented using descriptive statistics and nonparametric methods of analysis. The level of significance was set at $\mathrm{P}<0.05$. For the statistical analysis of data, SPSS software for Windows (edition 23.0; SPSS, Inc, IBM Company, Chicago, Illinois, USA) was used. Statistical analysis was carried out with diagrams, the non-parametric Kolmogorov-Smirnov method and the non-parametric Mann-Whitney and Wilcoxon methods.

\section{RESULTS}

\section{Study population}

Initial screening included 157 inpatients, which corresponded to 168 Candida positive blood cultures. Seven patients with endocarditis were excluded. Demographic data are presented in table 1.

The most common antifungal agents used for treatment were echinocandins (49.3\%, specifically: anidulafungin $18.7 \%$, micafungin $18.1 \%$, caspofungin $12.5 \%$ ), followed by azoles $(38.7 \%$, specifically: fluconazole $38.0 \%$ and voriconazole $0.7 \%$ ) and liposomal amphotericin B (12\%). Of the Candida species isolated, 42\% were C. albicans, 24.9\% C. parapsilosis, 13.1\% C. tropicalis, $8.3 \%$ C. glabrata, $4.9 \%$ C. krusei, 3.1\% C. lusitaniae, 1.4\% C. kefyr, 1.1\% C. lipolytica, 0.6\% C. intermedia and 0.6\% C. guillermondii (figure 1).

In an attempt to find a correlation between the consumption of echinocandins and amphotericin B and the MICs of each Candida species, we used DDDs of each year and the MICs of
Table 1 Demographic data

\begin{tabular}{ll}
\hline Age (years), mean (range) & $\mathbf{6 6 . 2 ( 1 8 - 9 3 )}$ \\
\hline Gender & Male: $62.5 \%$, female: $37.5 \%$ \\
\hline Comorbidities & $\begin{array}{l}\text { Cancer: } 28 \% \\
\text { Cardiovascular diseases: } 16.7 \% \\
\text { Neurological diseases: } 13.7 \%\end{array}$ \\
\hline Candidaemia per 100 bed-days & 0.037 \\
\hline Endocarditis & $4.45 \%$ \\
\hline Neutropenia & $9.6 \%$ \\
Chemotherapy/radiotherapy & $7.1 \%$ \\
\hline Intensive/intermediate care unit & $38.9 \%$ \\
\hline 28-Day mortality & $42.3 \%$ \\
\hline $\begin{array}{l}\text { Duration of treatment (days), } \\
\text { mean (range) }\end{array}$ & $18(9-25)$ \\
\hline
\end{tabular}

each species. Specifically for C. parapsilosis, DDDs of echinocandins followed a similar pattern to the corresponding average MICs as shown in table $2(\mathrm{P}=0.11)$.

\section{Analysis of costs}

The daily costs of each antifungal drug in Greece are shown in table 3 .

Of 92 patients treated with echinocandins or liposomal amphotericin B, 51 were eligible for de-escalation to fluconazole, but this was done in only 23 (figure 2). Of 28 patients who were eligible but treatment was not de-escalated, 19 were being treated with echinocandins and nine with liposomal amphotericin B. Additionally, nine patients initially treated with fluconazole were unnecessarily re-escalated to echinocandins. The drug cost for these 37 patients was $€ 294375$. If de-escalation had been implemented and unjustified re-escalation had not been carried out, the drug cost would have been $€ 82$ 538-that is, a saving of $€ 211837$ over 6 years or $€ 35306$ a year $(\mathrm{P}<0.05)$ (figure 3).

\section{DISCUSSION}

Although antimicrobial stewardship programmes are one of the highest priorities in healthcare systems and many articles have been published, few of them refer to the implementation of antifungal stewardship and highlight specific points on which efforts should be focused. In our 6-year retrospective study we examined the use of antifungal agents for confirmed candidaemia in hospitalised patients. In this study, candidaemia occurred more

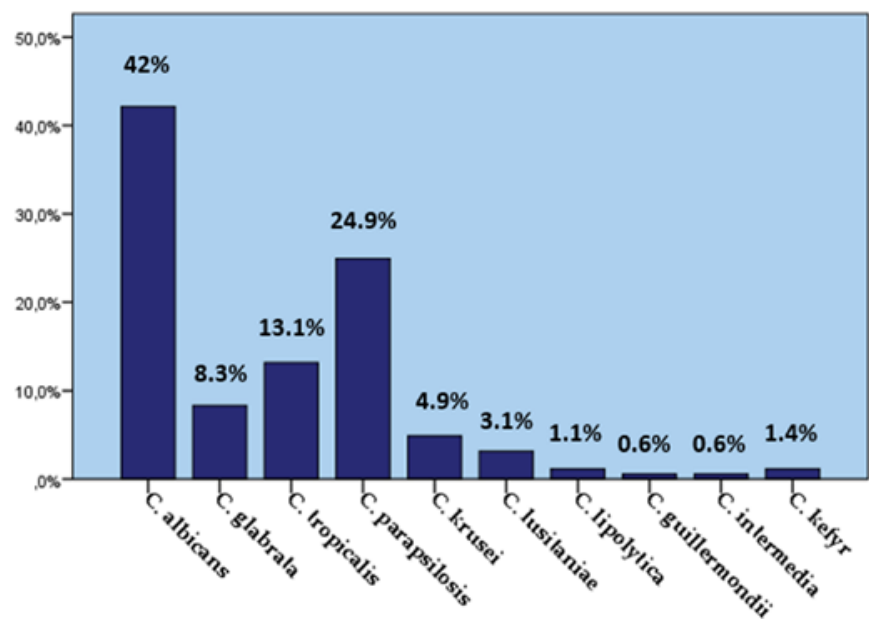

Figure 1 Isolated Candida species. 
Table 2 Presentation of defined daily does (DDDs) per 100 patientdays and the average minimum inhibitory concentrations (MICs) of caspofungin and micafungin for C. parapsilosis during 2013-2016

\begin{tabular}{lllll}
\hline & $\begin{array}{l}\text { DDDs per 100 } \\
\text { patient-days }\end{array}$ & MIC & MIC \\
Year & 2.12 & 0.88 & Micafungin \\
\hline 2013 & 1.81 & 0.527 & 0.66 \\
2014 & 1.79 & 0.71 & 0.62 \\
2015 & 2.29 & 0.77 & 0.62 \\
2016 & & & 0.65 \\
\hline
\end{tabular}

Table 3 Daily cost in euros of antifungal agents*

\begin{tabular}{lll}
\hline Drug* & Loading dose $(€)$ & Maintenance dose $(€)$ \\
\hline Fluconazole & 44 & 22 \\
\hline Voriconazole & 486.6 & 291.96 \\
Caspofungin & 425.35 & 332.05 \\
Anidulafungin & 651.3 & 325.65 \\
Micafungin & 312.6 & 312.6 \\
Liposomal & 761.11 & 761.11 \\
amphotericin B & & \\
\hline${ }^{*}$ Drug prices did not change significantly during the study.
\end{tabular}

often in elderly (mean age 66.2 years) male patients. Seven of the 157 patients examined were found to have endocarditis and excluded as management of treatment in these patients differed. ${ }^{2}$ Fifteen (9.6\%) patients were neutropenic and 61 (38.9\%) were admitted to the ICU (45) and the intermediate care unit (16).

The incidence of candidaemia is higher in critically ill patients, mainly owing to the presence of many risk factors such as central venous catheters, total parenteral nutrition, broad-spectrum antibiotics, high APACHE (Acute Physiology and Chronic Health Evaluation) scores and previous surgery. ${ }^{2}$ The mortality rate of candidaemia in our patients was $42.3 \%$, in accordance with another study, ${ }^{11}$ which showed a mortality rate of $45-50 \%$. In most cases, candidaemia occurred during hospitalisation and was not the major cause of admission. This finding is supported by other studies in which candidaemia occurred in hospitalised patients, such as elderly patients receiving corticosteroid treatment, patients with liver cirrhosis, with chronic obstructive pulmonary disease (COPD), patients receiving parenteral nutrition, or chemotherapy or monoclonal antibody treatment. ${ }^{12} 13$

Cancer was found to be the most common comorbidity, in accordance with other studies in Europe ${ }^{1415}$ and the findings of a Brazilian national surveillance programme. ${ }^{16}$ The Infectious Diseases Society of America (IDSA) guidelines emphasise the need for implementation of antifungal stewardship particularly in immunocompromised patients. ${ }^{17}$

In our study echinocandins were the most commonly used antifungal agents both in neutropenic and non-neutropenic patients, similar to the results published by Rajendran et al. ${ }^{18} 19$ The duration of treatment was 18 days, a finding confirmed by Pfaller et al. ${ }^{20}$ C. albicans was the most common species. The second most common cause of candidaemia was C. parapsilosis, as reported by other investigators for South Europe and Turkey. ${ }^{21} 22$ This is mirrored by an increase in the use of echinocandins which leads to higher MICs for C. parapsilosis. ${ }^{23}$ Quindós et al, ${ }^{21}$ found that the third most common cause was C. glabrata, whereas we found it to be C. tropicalis. Additionally, some rare Candida species, such as C. intermedia, C. guillermondii and C. kefyr were isolated. This increase in uncommon species is due to the increasing resistance to antifungal drugs. ${ }^{23}$ Another important point in this study was that C. krusei was the second most encountered Candida species in neutropenic patients, in accordance with the study by Arendrup and Perlin. ${ }^{4}$ Of the Candida species this leads to the highest mortality rates, while C. parapsilosis is less virulent than other Candida spp. ${ }^{20}$

As in the study of Van der Geest, treatment can be safely and effectively de-escalated to fluconazole in critically ill patients with invasive, fluconazole-susceptible C. albicans infections ${ }^{6}$, and another study found no difference between the efficacy and safety of echinocandins and fluconazole for the treatment of patients with fungal infections. De-escalation is also recommended by most recent fungal infections management guidelines. ${ }^{12}{ }^{24}$ In addition, fluconazole is a safe drug, which can be given orally with high bioavailability. It penetrates most body tissues, including the central nervous system (CNS) and urine, ${ }^{25}$ while echinocandins are not distributed in the $\mathrm{CNS}^{26}$ In order



Figure 2 Study flow chart demonstrating the use of antifungal agents. 


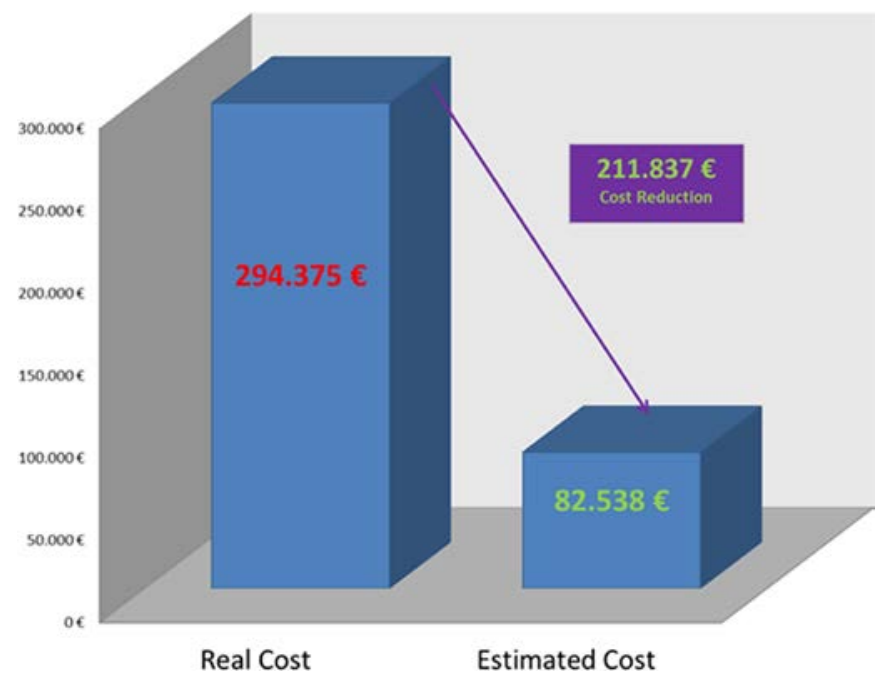

Figure 3 Estimated financial loss during the 6-year study.

to highlight the importance of de-escalation therapy, we must consider two important factors: (a) the emerging resistance to fluconazole and also to new potent antifungal agents such as echinocandins, and (b) the high cost of echinocandins or liposomal amphotericin B treatment.

Several studies, have indicated that resistance to echinocandins has increased. ${ }^{42728}$ These findings are in accordance with our results. Two strains of C. glabrata resistant to micafungin and caspofungin were found, which were also resistant to fluconazole, so de-escalation was not possible and the patients continued treatment with liposomal amphotericin B. Moreover, a resistant strain of C. albicans was reported. The patient did not belong to the group of patients eligible for step-down because he had repeated positive cultures. Detection of the two C. glabrata strains resistant to echinocandins is probably associated with the extended use of echinocandins throughout the hospitalisation of the patients. In addition, resistance of C. glabrata has been associated with factors such as previous exposure to echinocandin, a previous episode of candidaemia and fluconazole resistance. ${ }^{28}$

The resistance concerning C. glabrata is alarming, as it is a strain for which possible treatments are limited.

Resistance to C. glabrata was also found in a study conducted in Scotland, ${ }^{18}$ and by the study of Vallabhaneni et al. ${ }^{28}$ Having found the resistant strains, we also compared the trend of the average MICs for each Candida species with consumption by using DDDs per 100 patient-days. We observed a corresponding shift in the trends of the DDDs per 100 patient- days and the MICs of caspofungin and mycafungin for C. parapsilosis. Consequently, when use of echinocandins was reduced, the MICs for mycafungin and caspofungin were also reduced. For liposomal amphotericin B, no correlation between MICs and DDDs per 100 patient-days was found. We should add that we found a clear increase in the MIC of fluconazole for $C$. parapsilosis (1.08 to 6.45). This can be explained by the use of fluconazole as empirical or prophylactic therapy or by a possible cross-resistance with echinocadins. ${ }^{29}$

As mentioned above de-escalation of treatment is safe. In this study, we have found that $30.4 \%$ of the patients receiving echinocandins or liposomal amphotericin B could have been stepped down. Additionally, treatment was unjustifiably re-escalated in $9(15.5 \%)$ patients. This can be attributed to three reasons. First, most of those patients were in an ICU or or an intermediate care unit and the treating physician did not want to risk changing an effective drug in a clinically stable patient. Second, an infection control specialist was not part of the treating physician team and, finally, there was no multidisciplinary team (infection control specialist, clinical pharmacist) to control the de-escalation process. Not de-escalating from echinocandins or liposomal amphotericin B to fluconazole or unreasonably stepping-up led to significant costs for the hospital. A non-negligible annual cost of $€ 35306.05$ was estimated and found to be statistically significant. The two following studies had similar findings. Bal et al calculated a significant cost of $£ 1558.03$ per episode in the university hospitals Crosshouse and Ayr of England. ${ }^{30}$ This study was based on 37 patients for the years 2011-2013. Treatment for 19 of 27 patients was started with an echinocandin or voriconazole and changed to fluconazole. The second study was performed by Heimann et al at the University Hospital of Cologne, who found that the treatment with echinocandins resulted in excess costs of $€ 15690$ per patient. ${ }^{31}$

In conclusion, our study, in accordance with the findings of other studies, indicates the need for antifungal stewardship as part of an antimicrobial stewardship programme in hospitals. The presence of a multidisciplinary team, including an infection control specialist and a clinical pharmacist, would result in limits being placed on prescription of advanced antifungal agents. Moreover, this team would control the de-escalation process, if applicable, which would reduce costs and probably decrease the emergence of resistant Candida species. Finally, it is very important to highlight that this is not an epidemiological study and the results are not conclusive owing to the limited number of patients and cultures. Multicentre studies are needed.

\section{What this paper adds}

What is already known on this subject

- Many articles have been published on the appropriate use of antifungal agents, but few refer to the implementation of antifungal stewardship as part of an antimicrobial stewardship programme and highlight specific points on which efforts should be focused.

- Use of echinocandins and liposomal amphotericin B is associated with high costs.

\section{What this study adds}

- Our data indicate that empirical antifungal therapy is appropriate. However, the de-escalation process for targeted antifungal therapy is not implemented according to the guidelines.

- The presence of a multidisciplinary team, including an infection control specialist and a clinical pharmacist, would limit prescription of advanced antifungal agents for empirical therapy. Moreover, this team would control the de-escalation process, where applicable, leading to cost reduction and probably a decrease the emergence of resistant Candida species.

- Adoption of antifungal stewardship programmes in hospitals is essential.

Funding The authors have not declared a specific grant for this research from any funding agency in the public, commercial or not-for-profit sectors.

Competing interests None declared.

Patient consent Not required.

Provenance and peer review Not commissioned; externally peer reviewed. 
(C) European Association of Hospital Pharmacists (unless otherwise stated in the text of the article) 2020. All rights reserved. No commercial use is permitted unless otherwise expressly granted.

\section{REFERENCES}

1 Fridkin SK. The changing face of fungal infections in health care settings. Clin Infect Dis 2005;41:1455-60.

2 Pappas PG, Kauffman CA, Andes DR, et al. Executive summary: clinical practice guideline for the management of candidiasis: 2016 update by the Infectious Diseases Society of America. Clin Infect Dis 2016;62:409-17.

3 Kołaczkowska A, Kołaczkowski M. Drug resistance mechanisms and their regulation in non-albicans Candida species. J Antimicrob Chemother 2016;71:1438-50.

4 Arendrup MC, Perlin DS. Echinocandin resistance: an emerging clinical problem? Curr Opin Infect Dis 2014;27:484-92.

5 Grau S, Pozo JC, Romá E, et al. Cost-effectiveness of three echinocandins and fluconazole in the treatment of candidemia and/or invasive candidiasis in nonneutropenic adult patients. Clinicoecon Outcomes Res 2015;7:718-26.

6 van der Geest PJ, Rijnders BJ, Vonk AG, et al. Echinocandin to fluconazole step-down therapy in critically ill patients with invasive, susceptible Candida albicans infections. Mycoses 2016;59:179-85.

7 Falcone M, Concia E, lori l, et al. Identification and management of invasive mycoses in internal medicine: a road-map for physicians. Intern Emerg Med 2014;9:501-11.

8 Takesue Y, Ueda T, Mikamo H, et al. Management bundles for candidaemia: the impact of compliance on clinical outcomes. J Antimicrob Chemother 2015;70:587-93.

9 Valerio M, Rodriguez-Gonzalez CG, Muñoz P, et al. Evaluation of antifungal use in a tertiary care institution: antifungal stewardship urgently needed. J Antimicrob Chemother 2014;69:1993-9.

10 Fothergill AW, Sutton DA, McCarthy DI, et al. Impact of new antifungal breakpoints on antifungal resistance in Candida species. J Clin Microbiol 2014;52:994-7.

11 Yılmaz Karadağ F, Ergen P, Aydın Ö, et al. Evaluation of epidemiological characteristics and risk factors affecting mortality in patients with candidemia. Turk J Med SCi 2016;46:1724-8.

12 Antinori S, Milazzo L, Sollima S, et al. Candidemia and invasive candidiasis in adults: a narrative review. Eur J Intern Med 2016;34:21-8.

13 Delaloye J, Calandra T. Invasive candidiasis as a cause of sepsis in the critically ill patient. Virulence 2014;5:161-9.

14 Alfandari S, Berthon C, Coiteux V. Antifungal stewardship: Implementation in a French teaching hospital. Méd Mal Infect 2014;44:154-8.

15 Mondain V, Lieutier F, Hasseine L, et al. A 6-year antifungal stewardship programme in a teaching hospital. Infection 2013:41:621-8.
16 Doi AM, Pignatari AC, Edmond MB, et al. Epidemiology and microbiologic characterization of nosocomial candidemia from a Brazilian national surveillance program. PLoS One 2016;11:e0146909.

17 Barlam TF, Cosgrove SE, Abbo LM, et al. Implementing an antibiotic stewardship program: guidelines by the Infectious Diseases Society of America and the Society for Healthcare Epidemiology of America. Clin Infect Dis 2016;62:e51-77.

18 Rajendran R, Sherry L, Deshpande A, et al. A prospective surveillance study of candidaemia: epidemiology, risk factors, antifungal treatment and outcome in hospitalized patients. Front Microbiol 2016;7:915.

19 Campoy S, Adrio JL. Antifungals. Biochem Pharmacol 2017;133:86-96.

20 Pfaller M, Neofytos D, Diekema D, et al. Epidemiology and outcomes of candidemia in 3648 patients: data from the Prospective Antifungal Therapy (PATH Alliance $囚$ ) registry, 2004-2008. Diagn Microbiol Infect Dis 2012;74:323-31.

21 Quindós G. Epidemiology of candidaemia and invasive candidiasis. A changing face. Rev Iberoam Micol 2014;31:42-8.

22 Ulu Kilic A, Alp E, Cevahir F, et al. Epidemiology and cost implications of candidemia, a 6-year analysis from a developing country. Mycoses 2017;60:198-203.

23 Gonçalves SS, Souza ACR, Chowdhary A, et al. Epidemiology and molecular mechanisms of antifungal resistance in Candida and Aspergillus. Mycoses 2016;59:198-219.

24 Wang JF, Xue Y, Zhu XB, et al. Efficacy and safety of echinocandins versus triazoles for the prophylaxis and treatment of fungal infections: a meta-analysis of RCTs. Eur J Clin Microbiol Infect Dis 2015;34:651-9.

25 Nett JE, Andes DR. Antifungal agents: spectrum of activity, pharmacology, and clinical indications. Infect Dis Clin North Am 2016;30:51-83.

26 Aguilar-Zapata D, Petraitiene R, Petraitis V. Echinocandins: the expanding antifungal armamentarium. Clin Infect Dis 2015;61:S604-11.

27 Teo JQ, Candra SR, Lee SJ, et al. Candidemia in a major regional tertiary referral hospital - epidemiology, practice patterns and outcomes. Antimicrob Resist Infect Control 2017;11:27.

28 Vallabhaneni S, Cleveland AA, Farley MM. Epidemiology and risk factors for echinocandin nonsusceptible Candida glabrata bloodstream infections: data from a large multi-site population-based candidemia surveillance program, 2008-2014. Open Forum Infect Dis 2015;4:163.

29 Pham CD, Iqbal N, Bolden CB, et al. Role of FKS Mutations in Candida glabrata: MIC values, echinocandin resistance, and multidrug resistance. Antimicrob Agents Chemother 2014;58:4690-6.

30 Bal AM, Shankland GS, Scott G, et al. Antifungal step-down therapy based on hospital intravenous to oral switch policy and susceptibility testing in adult patients with candidaemia: a single centre experience. Int J Clin Pract 2014;68:20-7.

31 Heimann SM, Cornely OA, Wisplinghoff $\mathrm{H}$, et al. Candidemia in the intensive care unit: analysis of direct treatment costs and clinical outcome in patients treated with echinocandins or fluconazole. Eur J Clin Microbiol Infect Dis 2015:34:331-8. 